\title{
miR-3188 Regulates proliferation and apoptosis of granulosa cells by targeting KCNA5 in the polycystic ovary syndrome
}

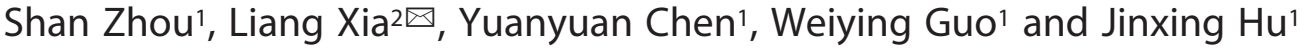 \\ 'Department of Endocrinology, Hwa Mei Hospital, University of Chinese Academy of Sciences, Ningbo Institute of Life and Health Industry, \\ University of Chinese Academy of Sciences, Ningbo City, Zhejiang Province, 315010, China; 2Department of Gynecology, Hwa Mei Hospital, \\ University of Chinese Academy of Sciences, Ningbo Institute of Life and Health Industry, University of Chinese Academy of Sciences, Ningbo \\ City, Zhejiang Province, 315010, China
}

\begin{abstract}
Abnormal proliferation of granulosa cells is implicated in ovarian dysfunction and dysregulated folliculogenesis in the polycystic ovary syndrome (PCOS). Aberrant microRNA (miRNA) expression might contribute to disordered folliculogenesis and granulosa cell proliferation in PCOS. This study aimed to investigate the roles of miR-3188 in ovarian dysfunction, as well as the mechanism involved in granulosa cell proliferation in PCOS. Firstly, peripheral blood samples were isolated from PCOS patients and healthy controls, and qRT-PCR analysis demonstrated a dramatic increase in miR-3188 in PCOS patients when compared to the healthy controls. Secondly, miR-3188 overexpression increased cell viability of the granulosalike tumor cell line (KGN). However, cell viability of KGN was repressed by interference with miR-3188. MiR-3188 promoted cell cycle of KGN through increasing cyclinD1 and decreasing p21 levels. Moreover, cell apoptosis was suppressed by miR-3188 in KGN, indicated by enhanced $\mathrm{BCl}-2$, and reduced Bax and cleaved caspase-3 levels, whereas knockdown of miR-3188 resulted in opposite effects. Lastly, potassium voltage-gated channel subfamily A member 5 (KCNA5) was verified as a target of miR3188. KCNA5 expression was decreased and displayed negative correlation with miR-3188 levels in PCOS patients. Overexpression of KCNA5 attenuated the promotive effects of miR-3188 on cell viability and cell cycle in KGN. In conclusion, miR-3188, a key miRNA enhanced in PCOS, promoted granulosa cell proliferation through down-regulation of KCNA5, providing a new therapeutic target for PCOS.
\end{abstract}

Keywords: miR-3188, KCNA5, proliferation, granulosa cell, PCOS Received: 03 August, 2020; revised: 24 September, 2020; accepted: 30 October, 2020; available on-line: 03 February, 2021

⿶e-mail: liangxia667@163.com

Abbreviations: KGN, granulosa-like tumor cell line; KCNA5, potassium voltage-gated channel subfamily A member 5; PCOS, polycystic ovary syndrome

\section{INTRODUCTION}

The polycystic ovary syndrome (PCOS) is a common heterogeneous endocrine disease, which could result in anovulation and infertility in women of childbearing age (Stanosz et al., 2018; Youssef, 2019). Ovarian biopsy in infertile PCOS patients showed increased accumulation of early follicles than in healthy women, suggesting that abnormal follicle development in the early stages may be the root cause of PCOS (Franks et al., 2008). Further study revealed that the growth rate of granulosa cells, which is critical for follicle maturation, was higher in PCOS patients than in healthy controls (Webber et al., 2007). Abnormal granulosa cell proliferation and differentiation are related to dysregulated folliculogenesis, thereby leading to the pathogenesis of PCOS (Stubbs et al., 2007). Therefore, regulation of follicular granulosa cell proliferation or apoptosis may be the direction of treatment for PCOS.

MiRNAs have been reported to be involved in granulosa cell function ( $\mathrm{Tu}$ et al., 2019), and the pathogenesis of PCOS (Sorensen et al., 2014). Reduction in miR-16 levels in PCOS could inhibit proliferation and promote apoptosis of ovarian granulosa cells (Fu et al., 2018). MiR-222 has been reported to promote proliferation and inhibit apoptosis of ovarian granulosa cells, thus promoting the progression of PCOS (Huang et al., 2019). MiR-3188 levels, with the ability to inhibit cell proliferation in non-small cell lung cancer (Wang et al., 2018) and head and neck cancer (Wang et al., 2019), were found to be increased in PCOS ovarian granulosa cells (Hou et al., 2019; Wang et al., 2019). Therefore, we hypothesized that miR-3188 might promote proliferation and inhibit apoptosis of ovarian granulosa cells during PCOS progression.

Potassium voltage-gated channel subfamily A member 5 (KCNA5) has been found to be associated with cell proliferation and apoptosis in various cell types (Ryland et al., 2015; Qu et al., 2018). Recently, KCNA5 has been shown to participate in miR-3940-5p-mediated granulosa cell proliferation in PCOS (Gao et al., 2020). However, whether or not miR-3188 could target KCNA5 to regulate granulosa cell proliferation in PCOS remains elusive.

In this study, the expression level of miR-3188 in granulosa cells of PCOS patients was examined. The role and mechanism of miR-3188-mediated granulosa cell proliferation were then evaluated. The findings from this study suggest that miR-3188 may be a promising therapeutic target for PCOS.

\section{MATERIALS AND METHODS}

\section{Patient samples}

This study was approved by the Ethics Committee of the Hwa Mei Hospital, University of Chinese Academy of Sciences. A total of 28 PCOS patients and 36 healthy controls with written informed consents were recruited from the Hwa Mei Hospital, University of Chinese Academy of Sciences. Peripheral blood samples were collected from the patients and controls into tubes (Pre- 
AnalytiX, Hombrechtikon, Switzerland). Samples were stored at $-80^{\circ} \mathrm{C}$ for subsequent analyses.

\section{Cell culture and transfection}

Granulosa-like tumor cell line (KGN), acquired from DSMZ (Braunschweig, Germany), was cultured in DMEM/F12 medium (Transgene, Beijing, China) containing 10\% fetal bovine serum (Sigma-Aldrich, St. Louis, MO, USA) at $37^{\circ} \mathrm{C}$ in an incubator. Full length of KCNA5 was amplified and cloned into pcDNA3.1 vector (Invitrogen, Carlsbad, CA, USA). MiR-3188 mimic, inhibitor and the corresponding negative controls (NC mimic, NC inhibitor) were synthesized by RiboBio (Guangzhou, China). KGN cells were transfected with the mimic, inhibitor or pcDNA vector using Lipofectamine 2000 (Invitrogen).

\section{Cell viability}

KGN cells $\left(2 \times 10^{3}\right.$ cells/well $)$ were seeded and cultured for 24 hours. Cells were incubated with $20 \mu \mathrm{L}$ of 5 $\mathrm{mg} / \mathrm{mL}$ MT'T solution (KeyGEN, Nanjing, China) for 4 hours before the measurement of absorbance at $490 \mathrm{~nm}$ using an Epoch microplate Reader (BioTek, Winooski, VT, USA).

\section{Flow cytometry}

KGN cells $\left(1 \times 10^{6}\right.$ cells $)$ were trypsin digested and harvested, and then stained with $5 \mu \mathrm{L}$ of $100 \mu \mathrm{g} / \mathrm{mL}$ propidium iodide after incubation with $1 \mathrm{U} / \mathrm{ml}$ of ribonuclease (Abcam, Cambridge, MA, USA). Cell cycle distribution was analyzed by the FACS flow cytometer (Attune, Life Technologies, Darmstadt, Germany). For cell apoptosis analysis, cells were incubated with fluorescein isothiocyanate-conjugated annexin $\mathrm{V}$ after staining with propidium iodide. Cell apoptosis distribution was also analyzed by the FACS flow cytometer.

\section{Luciferase reporter assay}

pGL3-basic vectors (Promega, Madison, WI, USA) containing wildtype or mutant 3'UTR of KCNA5 were constructed, and named KCNA5 wt and KCNA5 mut, respectively. pRL-TK, miR-3188 inhibitor or NC inhibitor, and KCNA5 wt or KCNA5 mut were cotransfected into KGN cells. The luciferase activities were measured 24 hours later by Luciferase Assay System (Promega).

\section{qRT-PCR}

A total of $1 \mu \mathrm{g}$ of RNA was extracted from granulosa cells or KGN by TRIzol (TIANGEN, Beijing, China), and reverse transcribed into cDNAs. qRT-PCR was conducted using a SYBR Green PCR kit (TransGen, Beijing, China). Primers used in this study are as follows: miR-3188: sense, 5'-AGAGGCTT'TGTGCGGATACGGG-3', antisense, 5'-TATGGAACGCTTCAC-3'; KCNA5: sense, 5'-GTAACGTCAAGGCCAAGAGC-3', antisense, 5'-TCCCAT'TCCCTACTCCACTG-3'; GAPDH: sense, 5'-AACGTGTCAGTOGTGGACCTG-3', antisense, 5'-AGTGGGTGTCGCTGTFGAAGT-3'; U6: sense, 5'-CTCGCTTTCGGCAGCACA-3', antisense, 5'-AACGCTTCACGAAT'T'TGCGT-3'.

\section{Western blotting}

Cell lysates were obtained from KGN using RIPA buffer, and then concentrated by using a bicinchoninic acid kit (Wuhan Boster Biological Technology, Hubei, China). A total of $30 \mu \mathrm{g}$ protein samples were separated by $10 \%$ SDS-PAGE, and electro-transferred onto polyvinylidene fluoride (PVDF) membranes (Bio-Rad, Hercules, CA, USA). After blocking in 5\% bovine serum albumin, membranes were immunoblotted with the following primary antibodies: KCNA5 (1:1500; Abcam, Cambridge, UK), cyclinD1 and p21 (1:2000; Abcam), Bcl-2, Bax and cleaved caspase-3 (1:2500; Abcam), and $\beta$-actin (1:3000; Abcam). Horseradish peroxidase-conjugated secondary antibody (1:5000; Abcam) was added before densitometry analysis with Pierce ECL Western Blotting Substrate (Pierce Biotechnology, Waltham, MA, USA).

\section{Statistical analysis}

Data are reported as mean \pm standard deviation. Statistical tests were conducted using SPSS 21.0 software (IBM-SPSS, Chicago, IL, USA). Student's $t$ test was used to compare differences between two groups. $p<0.05$ was considered as statistically significant.

\section{RESULTS}

\section{Enhanced miR-3188 level in PCOS increases granulosa cell viability}

To determine the expression of miR-3188 in PCOS, blood samples were collected from PCOS patients and healthy controls. qRT-PCR analysis demonstrated a significant increase in miR-3188 level in PCOS patients when compared to the controls $(p<0.01)$ (Fig. 1A). In order to establish the functional roles of miR-3188 in PCOS in vitro, KGN was transfected with miR-3188 mimic or inhibitor (Fig. 1B). The results of the MTT assay showed that miR-3188 overexpression increased cell viability of KGN, while miR-3188 knockdown led to opposite effects (Fig. 1C), suggesting the pro-proliferative role of miR-3188 in PCOS granulosa cells.

\section{Effect of miR-3188 on KGN cell cycle}

Cell cycles of KGN transfected with miR-3188 mimic or inhibitor were evaluated to assess the role of miR-3188 in PCOS progression. Results demonstrated that miR-3188 promoted cell cycle of KGN, with a decreased cell number in the G1 phase and increased cell number in the G2 and S phases (Fig. 2A). In contrast, in KGN cells transfected with miR-3188 inhibitor, the KGN cell number in the G1 phase was increased, whereas there was a lower number of cells in the G2 and $\mathrm{S}$ phase (Fig. 2A). This indicates that miR-3188 knockdown leads to cell cycle arrest in KGN. The levels of cell cycle proteins were also assessed by western blotting, which revealed that miR-3188 overexpression led to increased levels of cyclinD1 and reduced p21 to promote cell cycle progression (Fig. 2B). On the contrary, cyclin D1 level was reduced and that of p21 was increased in KGN transfected with miR-3188 inhibitor, consistent with cell cycle arrest (Fig. 2B). These results confirmed the pro-proliferative role of miR-3188 in PCOS granulosa cells.

\section{Effect of miR-3188 on KGN cell apoptosis}

Next, the effect of miR-3188 on cell apoptosis was investigated in $\mathrm{KGN}$, and the result indicated that miR-3188 suppressed cell apoptosis of KGN (Fig. $3 \mathrm{~A})$. Moreover, there was an increase in the number of apoptotic cells in KGN transfected with miR-3188 inhibitor (Fig. 3A), demonstrating the anti-apoptotic 
A

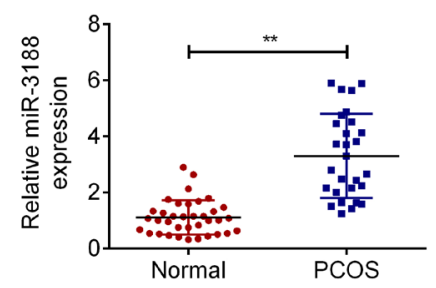

C

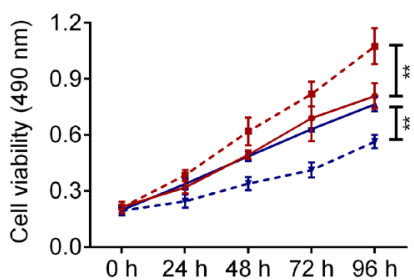

B

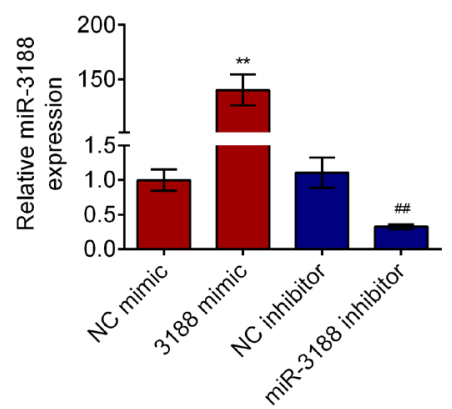

Figure 1. Enhanced miR-3188 level in PCOS increases granulosa cell viability

Expression level of miR-3188 in the peripheral blood samples of PCOS patients $(\mathrm{N}=28)$ and healthy controls $(\mathrm{N}=36)$. Transfection efficiency of miR-3188 mimic or inhibitor in KGN (N=3). Effect of miR-3188 on cell viability of $K G N(N=3) .{ }^{* *}, \# p<0.01$.

A
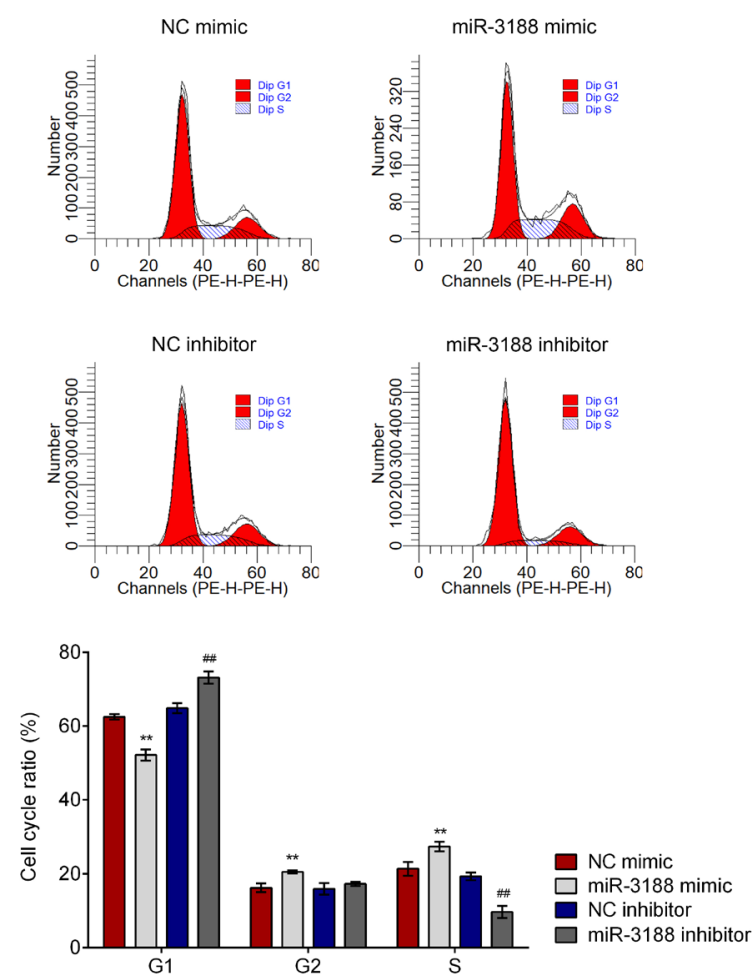

B
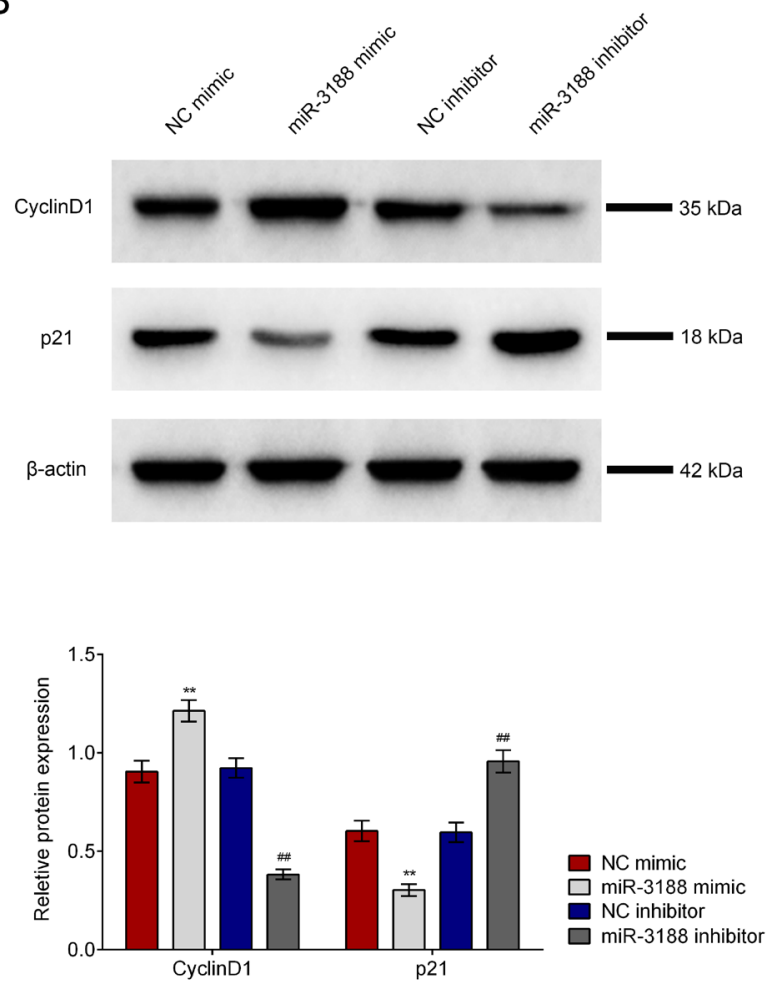

Figure 2. Effect of miR-3188 on cell cycle of KGN

Effect of miR-3188 on cell cycle of KGN (N=3). Effect of miR-3188 on the protein expression level of cyclin D1 and p21 in KGN (N=3). $* *, \# p<0.01$.

role of miR-3188 in granulosa cells. The levels of apoptotic proteins were also evaluated by western blotting. Results revealed that miR-3188 overexpression enhanced protein expression of $\mathrm{Bcl}-2$, but reduced the levels of Bax and cleaved caspase-3 (Fig. 3B). In contrast, Bax and cleaved caspase- 3 levels were enhanced, and $\mathrm{Bcl}-2$ level was reduced in KGN transfected with miR-3188 inhibitor (Fig. 3B). These results confirmed the anti-apoptotic role of miR-3188 in PCOS granulosa cells.

\section{miR-3188 negatively regulates KCNA5 in PCOS}

In order to uncover the underlying mechanisms of miR-3188 in the pathogenesis of PCOS, prediction of potential target genes of miR-3188 was performed using TargetScan (http://www.targetscan.org/vert_72/), followed by subsequent validation by luciferase reporter assays. The predicted binding sites between miR-3188 and $K C N A 5$, as well as mutated $K C N A 5$ binding site, which distupted the binding ability of $K C N A 5$ with miR-3188 are shown in Fig. 4A. Results from the lucif- 
A
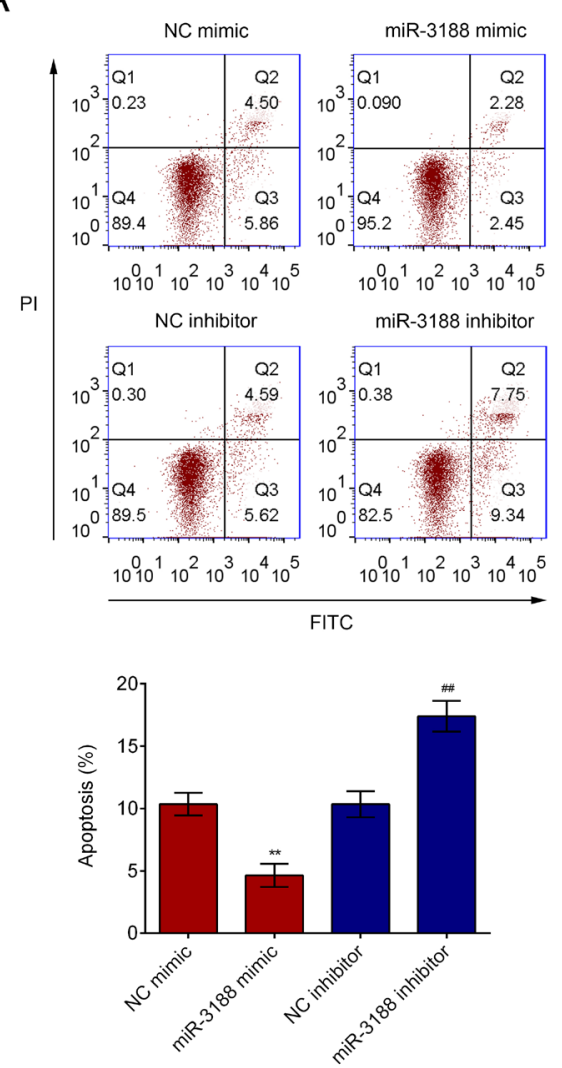

B
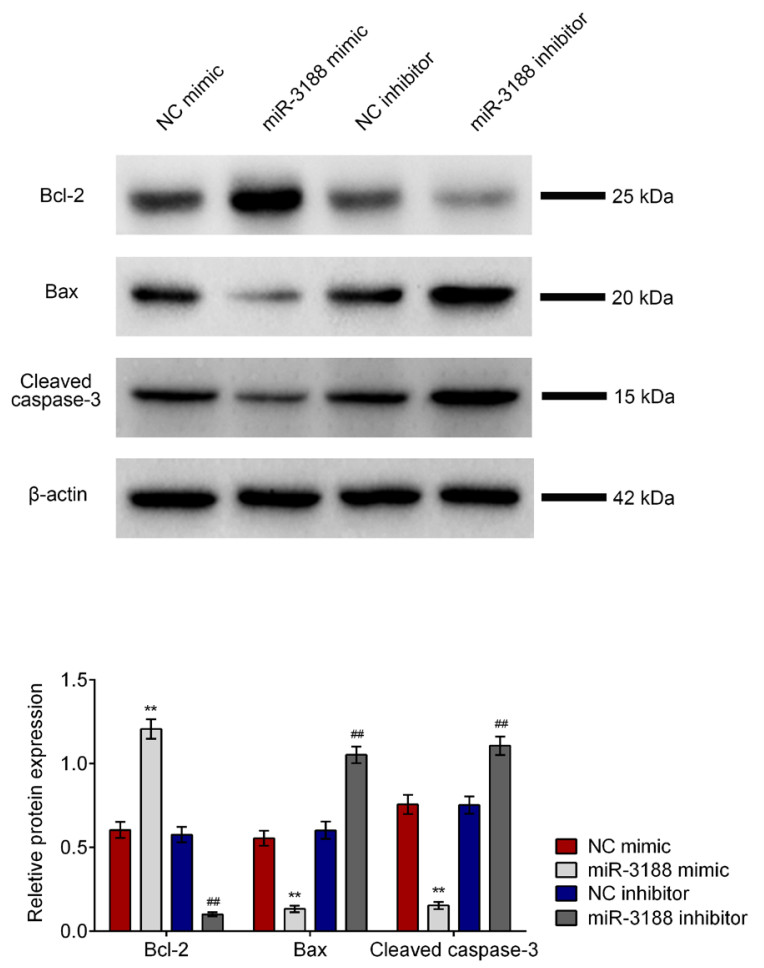

Figure 3. Effect of miR-3188 on cell apoptosis of KGN

Effect of miR-3188 on cell apoptosis of KGN ( $N=3$ ). Effect of miR-3188 on the protein expression level of Bcl-2, Bax and cleaved caspase-3 in $\mathrm{KGN}(\mathrm{N}=3)$. ${ }^{* *}, \# p<0.01$.

A

Position 587-593 of KCNA5 3' UTR

KCNA5 wt 5'...GUUUCCUGUGUCUCCAAGCCUCU....'
KCNA5 mut $\quad$ '...GUUUCCUGUGUCUCCUUCGGAGU...3'

C
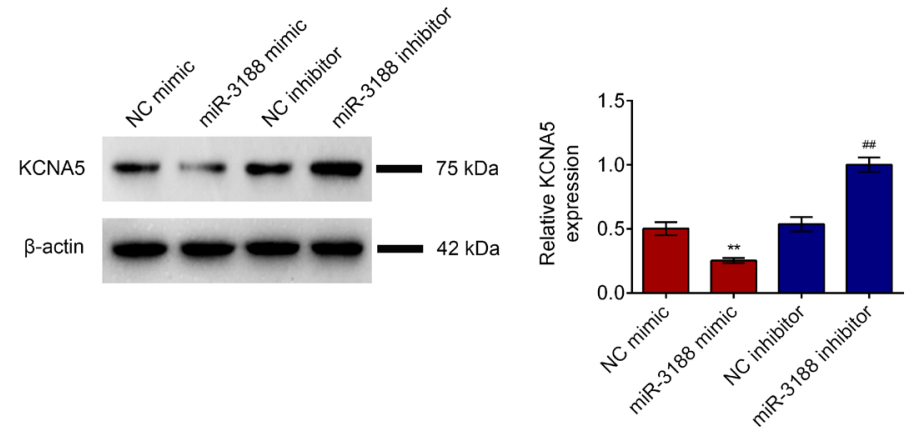

B

D

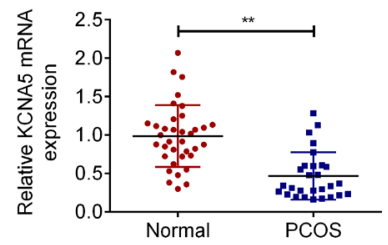

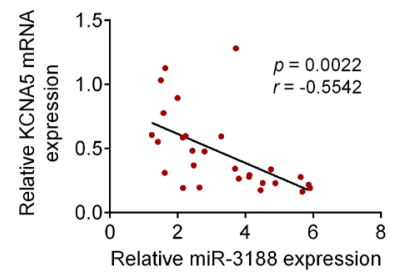

Figure 4. miR-3188 negatively regulates KCNA5

Potential binding site between miR-3188 and KCNA5, and KCNA5 mutant binding site. Effect of miR-3188 on luciferase activities of KCNA5 wt and KCNA5 mut in KGN (N=3). Effect of miR-3188 on the protein expression of KCNA5 in KGN ( $\mathrm{N}=3$ ). Expression level of KCNA5 in peripheral blood samples of PCOS patients $(\mathrm{N}=28)$ and healthy controls $(\mathrm{N}=36)$. Negative correlation between $\mathrm{KCNA5}$ and miR3188 in PCOS patients. ${ }^{* *}, \# \#<0.01$.

erase activity assays showed that transfection with miR3188 inhibitor increased luciferase activities of KCNA5 wt compared to NC inhibitor (Fig. 4B), while KCNA5 mut demonstrated no response to miR-3188 inhibitor or
NC inhibitor (Fig. 4B). Furthermore, the protein expression of KCNA5 was increased in KGN transfected with miR-3188 inhibitor, while it was decreased by miR-3188 mimic (Fig. 4C), confirming that KCNA5 was regulated 
A
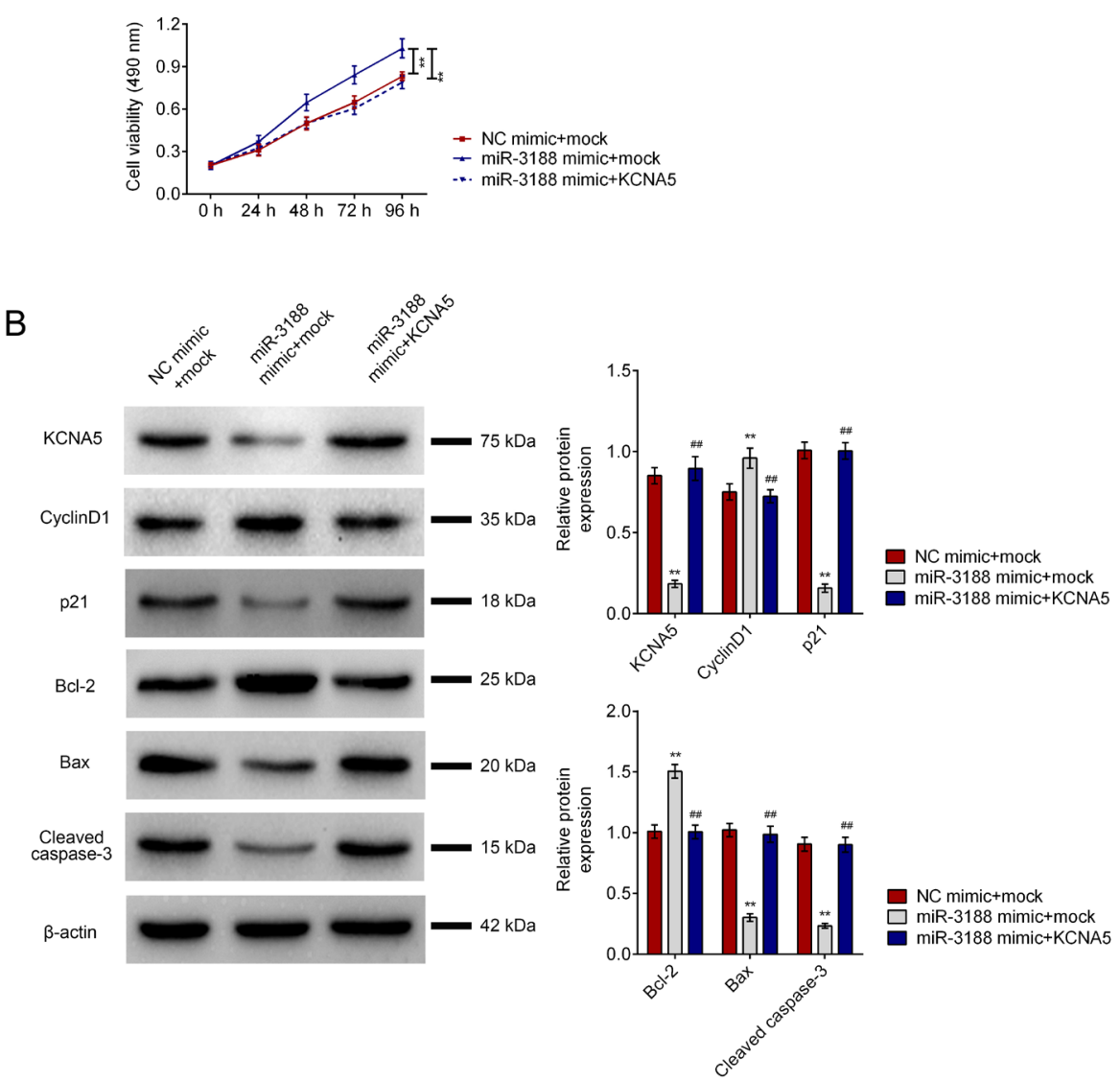

Figure 5. Overexpression of KCNA5 counteracts the promotive role of miR-3188 in PCOS progression

Effect of miR-3188 and KCNA5 on cell viability of KGN (N=3). Effect of miR-3188 and KCNA5 on the protein expression level of KCNA5, cyclin D1, p21, Bcl-2, Bax and cleaved caspase in KGN (N=3). ${ }^{* * * \# \#<0.01 .}$

by miR-3188 in granulosa cells. A significant decrease in KCNA5 was found in PCOS patients when compared to controls $(p<0.01)$ (Fig. 4D), and the expression of KCNA5 and miR-3188 revealed a negative correlation in PCOS patients (Fig. 4D). These results demonstrated that miR-3188 negatively regulates KCNA5 in PCOS.

\section{Over-expression of KCNA5 counteracts the promotive effects of miR-3188 on PCOS progression}

To explore the role of miR-3188/KCNA5 axis in PCOS granulosa cells, KGN was cotransfected with pcDNA-KCNA5 and miR-3188 mimic. KCNA5 overexpression attenuated miR-3188 mimic-mediated cell viability increase (Fig. 5A). Of note, miR-3188 mimic transfection failed to decrease the protein expression of KCNA5 in KCNA5-overexpressed cells (Fig. 5B). In addition, KCNA5 overexpression also lessened the miR-3188 overexpression-induced increase in cyclinD1 and Bcl-2 levels, and decrease in p21, Bax and cleaved caspase levels in KGN (Fig. 5B), further suggesting that miR-3188 could promote cell viability of granulosa cells in PCOS through down-regulation of KCNA5.

\section{DISCUSSION}

PCOS, characterized by such inconvenient symptoms as irregular menstrual bleeding and infertility, has been regarded as a "nuisance disease" devoid of effective therapeutic strategies (Conway et al., 2014; Peng et al., 2020). Inhibition of granulosa cell survival provides a novel insight into PCOS treatment (Zhong et al., 2018; Cox et al., 2020). Considering the important regulatory role of miRNAs in granulosa cell functions, miRNAs have been regarded as critical mediators in physiological and pathological conditions of PCOS (Tu et al., 2019). To date, miR-3188 has been shown as either suppressor or promoter in various tumors to regulate cell proliferation and apoptosis. The study presented here was performed to uncover the role of miR-3188 in granulosa cell proliferation and apoptosis in PCOS.

Biochemical markers, such as the luteinizing hormone or follicle-stimulating hormone levels, circulating androgens and hyperinsulinemia, are diagnostic criteria for PCOS (Barthelmess \& Naz 2014; EBERSOLE \& BONNY 2020). MiRNAs are also considered as diagnostic or prognostic biomarkers for PCOS (Wang et al., 2019). Here, in line with previous reports (Hou et al., 2019; Wang et al., 2019), miR-3188 was found to be increased in the peripheral blood samples of PCOS patients when compared to the healthy controls. However, to further validate the potential of miR-3188 as a diagnostic or prognostic marker for PCOS, the correlation between clinical features of PCOS patients and miR-3188 levels should be investigated in a future study.

Aberrant profiling of miRNAs that participated in steroidogenesis or the cell apoptosis process was detected in follicular fluid or granulosa cells in PCOS patients (Sorensen et al., 2014; Lionett et al., 2020). MiR-93, which has the ability to promote granulosa cell 


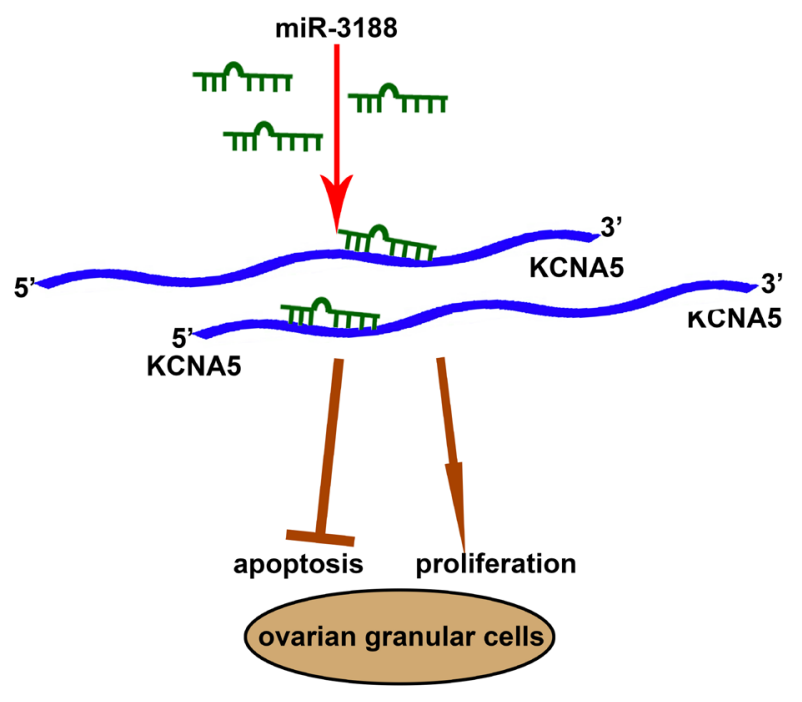

Figure 6. Graphic abstract shows the miR-3188/KCNA5 axis in proliferation and apoptosis of ovarian granulosa cells.

proliferation, could be a therapeutic target for PCOS (Jiang et al., 2015). Here, based on MTT, flow cytometry and western blotting analyses, our findings demonstrated that miR-3188 could increase granulosa cell viability, promote cell cycle progression through increasing cyclin D1 and decreasing p21 levels, and suppress cell apoptosis via promoting expression of $\mathrm{Bcl}-2$ and lowering Bax and cleaved caspase- 3 levels. Collectively, our results demonstrated that miR-3188 is a novel key miRNA that promotes granulosa cell proliferation in PCOS. This is in contrast to previous finding, which proposed that miR-3188 suppresses cell proliferation and cell cycle progression in non-small cell lung cancer cells through targeting mammalian target of rapamycin (Wang et al., 2018). This prompted the identification of cell type-specific downstream target of miR-3188 in PCOS granulosa cells.

Cation channels have been implicated in the apoptosis of granulosa cells, indicating their role in the etiology of PCOS (Köse \& Nazıroğlu 2015). Antagonists of potassium channel regulate granulosa cell proliferation and apoptosis (Manikkam et al., 2002; Traut et al., 2009). Previous study has shown that KCNA in granulosa cells binds to an antagonist, 4-aminopyridine, and decreases progesterone production through the steroidogenic pathway (Li et al., 2003). Here, potassium channel KCNA5 level was found to be reduced in granulosa cells of PCOS, and was validated to be the downstream target gene of miR-3188. Gao et al (Gao et al., 2020) has reported that KCNA5 is involved in miR-3940-5p-promoted granulosa cell proliferation in PCOS. Our functional assays revealed that overexpression of KCNA5 counteracted the promotive role of miR-3188 in granulosa cell viability and cell cycle progression, as well as the suppressive role of miR-3188 in granulosa cell apoptosis. MiRNAs could target steroid receptors or steroid synthesis enzymes involved in PCOS (Sorensen et al., 2014), and antagonists of KCNA could mediate progesterone accumulation in the granulosa cells ( $\mathrm{Li}$ et al., 2003). The role of miR$3188 / \mathrm{KCNA}$ in the steroidogenic pathway of granulosa cells should be investigated in a future study. Additionally, signaling pathways, including transforming growth factor- $\beta$ or follicle-stimulating hormones' pathway, which are known to be associated with miRNAs-medi- ated granulosa cell homeostasis (Tu et al., 2019), as well as pathways involved in miR-3188/KCNA-modulated granulosa cell proliferation in PCOS should also be investigated.

\section{CONCLUSION}

In conclusion, as shown in Fig. 6, our results indicated that increased miR-3188 levels in PCOS patients could promote cell viability and cell cycle progression, and suppress cell apoptosis through down-regulation of KCNA5. These observations provide novel insights into the potential development of miR-3188 as a therapeutic approach for the treatment of PCOS.

\section{Acknowledgements}

Not applicable.

\section{Funding}

This work was supported by the Hwa Mei Research Fund of the Hwa Mei Hospital, University of Chinese Academy of Sciences (Grant No. 2019HMKY32).

\section{Competing interests}

The authors state that there are no conflicts of interest to disclose.

\section{Ethics approval}

Ethical approval was obtained from the Ethics Committee of the Hwa Mei Hospital, University of Chinese Academy of Sciences.

\section{Statement of Informed Consent}

Written informed consent was obtained from a legally authorized representative(s) for anonymized patient information to be published in this article.

\section{Availability of data and materials}

All data generated or analyzed during this study are included in this published article.

\section{Authors' contributions}

Shan Zhou and Liang Xia designed the study, supervised the data collection, analyzed the data, Yuanyuan Chen interpreted the data and prepare the manuscript for publication, Weiying Guo and Jinxing $\mathrm{Hu}$ supervised the data collection, analyzed the data and reviewed the draft of the manuscript. All authors have read and approved the manuscript.

\section{REFERENCES}

Barthelmess EK, Naz RK (2014) Polycystic ovary syndrome: current status and future perspective. Front Biosci (Elite Ed) 6: 104-119. https://doi.org/10.2741/e695

Conway G, Dewailly D, Diamanti-Kandarakis E, Escobar-Morreale HF, Franks S, Gambineri A, Kelestimur F, Macut D, Micic D, Pasquali R, Pfeifer M, Pignatelli D, Pugeat M, Yildiz BO, EPSI Group (2014) The polycystic ovary syndrome: a position statement from the European Society of Endocrinology. Eur J Endocrinol 171: P1-29. https://doi.org/10.1530/EJE-14-0253

Cox MJ, Edwards MC, Rodriguez Paris V, Aflatounian A, Ledger WL, Gilchrist RB, Padmanabhan V, Handelsman DJ, Walters KA (2020) Androgen action in adipose tissue and the brain are key mediators in the development of PCOS traits in a mouse model. Endocrinology 161 (7). https://doi.org/10.1210/endocr/bqaa061 
Ebersole AM, Bonny AE (2020) Diagnosis and treatment of polycystic ovary syndrome in adolescent females. Clin Obstet Gynecol 63: 544 552. https://doi.org/10.1097/GRF.0000000000000538

Franks S, Stark J, Hardy K (2008) Follicle dynamics and anovulation in polycystic ovary syndrome. Hum Reprod Update 14: 367-378. https:// doi.org/10.1093/humupd/dmn015

Fu X, He Y, Wang X, Peng D, Chen X, Li X, Wan Q (2018) MicroRNA-16 promotes ovarian granulosa cell proliferation and suppresses apoptosis through targeting PDCD4 in polycystic ovarian syndrome. Cell Physiol Biochem 48: 670-682. https://doi.org/10.1159/000491894

Gao L, Wu D, Wu Y, Yang Z, Sheng J, Lin X, Huang H (2020) MiR3940-5p promotes granulosa cell proliferation through targeting KCNA5 in polycystic ovarian syndrome. Biochem Biophys Res Commun 524: 791-797. https://doi.org/10.1016/j.bbrc.2020.01.046

Hou Y, Wang Y, Xu S, Qi G, Wu X (2019) Bioinformatics identification of microRNAs involved in polycystic ovary syndrome based on microarray data. Mol Med Rep 20: 281-291. https://doi. org/10.3892/mmr.2019.10253

Huang X, She L, Luo X, Huang S, Wu J (2019) MiR-222 promotes the progression of polycystic ovary syndrome by targeting p27 Kip1. Pathol Res Pract 215: 918-923. https://doi.org/10.1016/j. prp.2019.01.038

Jiang L, Huang J, Li L, Chen Y, Chen X, Zhao X, Yang D (2015) MicroRNA-93 promotes ovarian granulosa cells proliferation through targeting CDKN1A in polycystic ovarian syndrome. J Clin Endocrinol Metabol 100: E729-E738. https://doi.org/10.1210/jc.2014-3827

Köse S, Nazıroğlu M (2015) N-acetyl cysteine reduces oxidative toxicity, apoptosis, and calcium entry through TRPV1 channels in the neutrophils of patients with polycystic ovary syndrome. Free Radic Res 49: 1-9. https://doi.org/10.3109/10715762.2015.1006214

Li Y, Ganta S, von Stein FB, Mason DE, Mitchell BM, Freeman LC (2003) 4-aminopyridine decreases progesterone production by porcine granulosa cells. Reprod Biol Endocrinol 1: 31. https://doi. org/10.1186/1477-7827-1-31

Lionett S, Kiel IA, Camera DM, Vanky E, Parr EB, Lydersen S, Hawley JA, Moholdt T (2020) Circulating and adipose tissue miRNAs in women with polycystic ovary syndrome and responses to high-intensity interval training. Front Physiol 11: 904. https://doi. org/ 10.3389 / fphys.2020.00904. eCollection 2020

Manikkam M, Y Li, BM Mitchell, DE Mason,LC Freeman (2002) Potassium channel antagonists influence porcine granulosa cell proliferation, differentiation, and apoptosis1. Biol Reprod 67: 88-98. https://doi.org/10.1095/biolreprod67.1.88

Peng Y, Guo L, Gu A, Shi B, Ren Y, Cong J, Yang X (2020) Electroacupuncture alleviates polycystic ovary syndrome-like symptoms through improving insulin resistance, mitochondrial dysfunction, and endoplasmic reticulum stress via enhancing autophagy in rats. Mol Med 26: 1-13. https://doi.org/10.1186/s10020-020-00198-8

Qu C, Sun J, Liu Y, Wang X, Wang L, Han C, Chen Q, Guan T, Li H, Zhang Y, Wang Y, Liu J, Zou W, Liu J (2018) Caveolin-1 facil- itated KCNA5 expression, promoting breast cancer viability. Oncol Lett 16: 4829-4838. https://doi.org/10.3892/ol.2018.9261

Ryland KE, Svoboda LK, Vesely ED, McIntyre JC, Zhang L, Martens JR, Lawlor ER (2015) Polycomb-dependent repression of the potassium channel-encoding gene KCNA5 promotes cancer cell survival under conditions of stress. Oncogene 34: 4591-4600. https://doi. org/10.1038/onc.2014.384

Sorensen AE, Wissing ML, Salo S, Englund AL, Dalgaard LT (2014) MicroRNAs Related to Polycystic Ovary Syndrome (PCOS). Genes (Basel) 5: 684-708. https://doi.org/10.3390/genes5030684

Stanosz S, von Mach Szczypiński J, Fogel A, Sowińska-Przepiera E, Kościuszkiewicz J, Żochowska E, Syrenicz A (2018) Concentration of serotonin in perimenopausal women with ductal breast cancer and polycystic ovary syndrome. Eur J Gynaecol Oncol 39: 884-888. https://ejgo.imrpress.com/EN/Y2018/V39/I6/884

Stubbs SA, Stark J, Dilworth SM, Franks S, Hardy K (2007) Abnormal preantral folliculogenesis in polycystic ovaries is associated with increased granulosa cell division. I Clin Endocrinol Metab 92: 44184426. https://doi.org/10.1210/jc.2007-0729

Traut MH, Berg D, Berg U, Mayerhofer A, Kunz L (2009) Identification and characterization of $\mathrm{Ca}^{2+}$-activated $\mathrm{K}+$ channels in granulosa cells of the human ovary. Reprod Biol Endocrinol 7: 28.

Tu J, Cheung AH-H, Chan CL-K, Chan W-Y (2019) The role of microRNAs in ovarian granulosa cells in health and disease. Front Endocrinol (Lausanne) 10: 174-174. https://doi.org/10.3389/fendo.2019.00174

Wang C, Liu E, Li W, Cui J, Li T (2018) MiR-3188 inhibits nonsmall cell lung cancer cell proliferation through FOXO1-mediated mTOR-p-PI3K/AKT-c-JUN signaling pathway. Front Pharmacol 9: 1362. https://doi.org/10.3389/fphar.2018.01362

Wang X, Qin X, Yan M, Shi J, Xu Q, Li Z, Yang W, Zhang J, Chen W (2019) Loss of exosomal miR-3188 in cancer-associated fibroblasts contributes to HNC progression. I Exp Clin Cancer Res 38: 151. https://doi.org/10.1186/s13046-019-1144-9

Wang Y, Xu S, Wang Y, Qi G, Hou Y, Sun C, Wu X (2019) Identification and potential value of candidate microRNAs in granulosa cells of polycystic ovary syndrome. Technol Health Care 27: 579-587. https://doi.org/10.3233/THC-181510

Webber L, Stubbs S, Stark J, Margara R, Trew G, Lavery S, Hardy K, Franks S (2007) Prolonged survival in culture of preantral follicles from polycystic ovaries. J Clin Endocrinol Metabol 92: 1975-1978. https://doi.org/10.1210/jc.2006-1422

Youssef HMG, Marei ESM, Rashed LA (2019) Long non-coding RNA steroid receptor activator in polycystic ovary syndrome: possible association with metabolic syndrome. Clini Exp Obstetrics Gynecol 46: 757-762. https://doi.org/10.12891/ ceog4774.2019

Zhong Z, Li F, Li Y, Qin S, Wen C, Fu Y, Xiao Q (2018) Inhibition of microRNA-19b promotes ovarian granulosa cell proliferation by targeting IGF-1 in polycystic ovary syndrome. Mol Med Rep 17: 4889-4898. https://doi.org/10.3892/mmr.2018.8463 